Research Article

\title{
Comparison of Saddle-Shaped Region of Head-Flow Curve between Axial-Flow Pump and Its Corresponding Axial-Flow Pump Device
}

\author{
Dongtao Ji $\mathbb{D}^{1}{ }^{1}$ Weigang Lu $\mathbb{D}^{1},{ }^{1}$ Linguang Lu, ${ }^{1}$ Lei Xu, ${ }^{1}$ Jun Liu, ${ }^{2}$ Wei Shi, ${ }^{2}$ and Ye Zhu ${ }^{1}$ \\ ${ }^{1}$ College of Hydraulic Science and Engineering, Yangzhou University, Yangzhou 225000, Jiangsu, China \\ ${ }^{2}$ Jiangsu Water Supply Co., Ltd., In Eastern Route of S-to-N Water Diversion Project, Nanjing 210019, Jiangsu, China \\ Correspondence should be addressed to Weigang Lu; wglu@yzu.edu.cn
}

Received 2 September 2021; Revised 3 November 2021; Accepted 15 November 2021; Published 29 November 2021

Academic Editor: Antonio Giuffrida

Copyright (c) 2021 Dongtao Ji et al. This is an open access article distributed under the Creative Commons Attribution License, which permits unrestricted use, distribution, and reproduction in any medium, provided the original work is properly cited.

In engineering, the highest operating head of the pumping station is usually controlled to be slightly lower than the lowest saddle bottom head of the axial-flow pump. However, in the practical operation, it is found that the highest operating head of the pumping station is obviously lower than the saddle bottom head of the pump device, which leads to the reduction of the operating range of the pumping station. To investigate the difference of lowest saddle bottom head between axial flow pump and axial flow pump device and apply it correctly, the energy performance tests of the TJ04-ZL-06 hydraulic model and its corresponding pump device were carried out to obtain the external curves, and numerical simulation was carried out to analyze and compare the internal flow field and pressure distribution. The results show that when the flow rate decreases, the first saddle-shaped region of the axial-flow pump and the saddle-shaped region of the pump device are caused by the decrease of the lift coefficient due to the increase of the attack angle between flow and blade. When the flow rate is less than $0.32 Q_{d}$, the influence range of backflow in the inlet pipe is large, which leads to the high-pressure zone near the wall of the inlet pressure measurement section during the pump performance test, and hence the second saddle-shaped region of the axial-flow pump is essentially a measurement illusion. It is suggested that the inlet pressure measurement section should be set at least $4 D_{p}$ away from the inlet flange of the impeller when testing the performance of the axial-flow pump under the condition of small flow rate, and the first saddle bottom head of the axial-flow pump or the saddle bottom head of the corresponding pump device can be considered as the control value of the highest head of the pumping station.

\section{Introduction}

Axial-flow pumps play significant roles in irrigation, urban water supply, and flood control [1-3]. In the design of pumping stations, to maximize the pumping station's efficiency and save energy, the pump must be equipped with an inlet and outlet passage to form a pump device. At present, in addition to a large number of studies on the operation of the axial-flow pump and axial-flow pump device under optimal conditions [4-7], some scholars have studied the operation and harm in the saddle-shaped region. It is found that there is vibration and noise when the axial-flow pump works in saddle-shaped region, which reduces the life of the pump and affects the safety of the pumping station [8-10].
Therefore, when selecting a pump for an axial-flow pumping station, the highest head of the pumping station must be lower than the lowest saddle bottom head of the axial-flow pump [11]. However, it is found in practical engineering that the highest head of the pumping station is much lower than the saddle bottom head of the pump device, which leads to the reduction of the operating range of the pumping station. The $Q-H$ curves of a large number of axial-flow pumps and their corresponding pump devices are compared, and it is found that there are two saddle-shaped regions of the axialflow pump [12], while there is only one saddle-shaped region of the pump device [13]. Typical $Q-H$ curves of the axial-flow pump and axial-flow pump device are shown in Figure 1. In addition, the first saddle bottom head of the pump is close to 


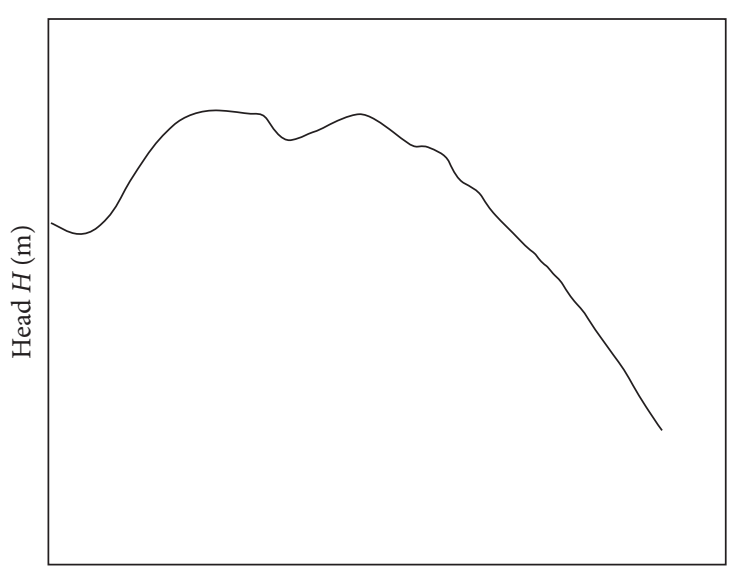

Discharge $Q\left(\mathrm{~L} \cdot \mathrm{s}^{-1}\right)$

(a)

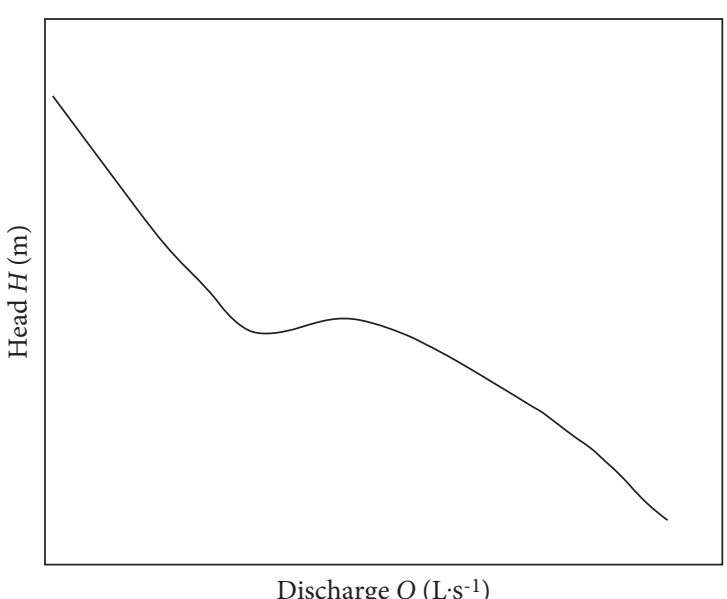

(b)

Figure 1: Head-flow curve: (a) axial-flow pump; (b) axial-flow pump device.

the saddle bottom head of the corresponding pump device and is obviously higher than the second saddle bottom head.

At present, there are some research studies on the operation of the axial-flow pump and pump device in saddleshaped region, but there is no study on the difference between the saddle-shaped region in $Q-H$ curves of the axial-flow pump and its corresponding pump device. Yang et al. [12] conducted a model test of the axial-flow pump, found that there are two saddle-shaped regions in the head curve, and measured the flow field with PIV technology. The results showed that when the flow rate decreases to $0.55 Q_{d}$, backflow starts to appear at the impeller inlet, and water flows out from the rim of the impeller. Zheng et al. [13] carried out a CFD analysis of the axial-flow pump device and found that a saddle-shaped region exists at $50 \% \sim 65 \% Q_{d}$. The axial-flow pump cannot operate stably under this condition. Goltz et al. [14] found that the impeller starts to stall when an inflection point occurs in the head curve by carrying out a test of hydraulic characteristics of a pump and captured some characteristics of the initial stall. Cheng et al. [15] conducted a CFD analysis of the pump device. The results showed that under small flow rate working condition, there is a large range of spiral backflow in the inlet passage. Wang et al. [16] found that the flow pattern in the impeller is well in the optimal condition. When the flow rate decreases to the saddle-shaped region, there is backflow at the impeller inlet.

Model test and numerical simulation are used in this study to investigate the cause of the difference between the saddleshaped region of the axial-flow pump and its corresponding pump device. This study can provide suggestions for the axialflow pump selection of large- and medium-sized pumping stations and the performance test of the axial-flow pump; the inlet pressure measurement section should be set at least $4 \mathrm{Dp}$ away from the inlet flange of impeller when testing the performance of axial flow pump under the condition of small flow rate; and the first saddle bottom head of axial flow pump or the saddle bottom head of corresponding pump device can be considered as the control value of the highest head of pumping station. This study has theoretical value and application value.

\section{Model Test}

2.1. Research Object. The inlet passage is the connection between the forebay and the impeller chamber. The purpose is to make the flow uniform and smooth and provide a good inlet condition for the impeller. The outlet passage is commonly used to recover kinetic energy [17]. The object of this study is an axial-flow pump (TJ04-ZL-06) and its corresponding shaft tubular pump device. The axial-flow pump has 3 blades and 6 guide vanes. The corresponding device consists of the aforementioned axial-flow pump, shaft inlet passage, and straight outlet passage.

The design requirements of the axial-flow pump are presented as follows: the impeller diameter $D=300 \mathrm{~mm}$ and the rotation speed $n=1450 \mathrm{r} / \mathrm{min}$. The tip clearance of model pump is within $0.2 \mathrm{~mm}$.

2.2. Experimental Setup. According to standards (e.g., SL140-2006, ISO 9906:2012, and ANSI/HI 14.6-2011), for the axial-flow pump model test, the inlet and outlet pressure measurement sections are set at twice the pipe diameter of the inlet and outlet flanges of the axial-flow pump, respectively, and the pipe diameter $D_{p}=350 \mathrm{~mm}$. For the pump device model test, the inlet pressure measurement section is arranged at the inlet section of the shaft inlet passage, whereas the outlet pressure measurement section is arranged at the outlet of the straight outlet passage. Four measurement points are arranged on each measurement section; for the circular section, points are set at the ends of two vertical diameters, whereas for the rectangular section, points are set at the midpoints of four edges. The model diagram of the experiment is shown in Figure 2. The location of the pressure measurement sections is shown in Figure 3.

2.3. Analysis of the Test. To ensure the comparability of the results of the two model tests, both model tests were conducted on the general hydraulic model test bench of the China Water Resources Beifang Co., Ltd. The general 


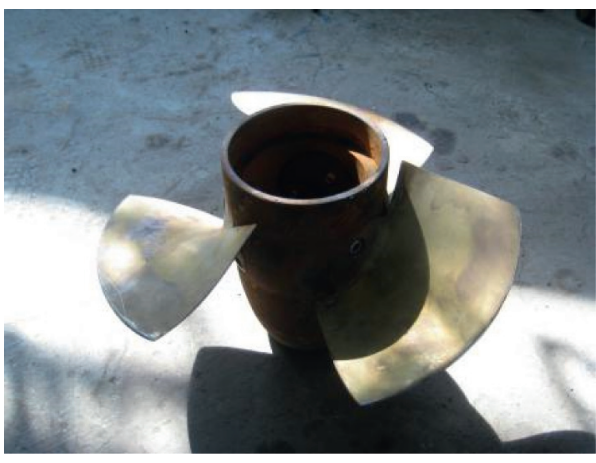

(a)

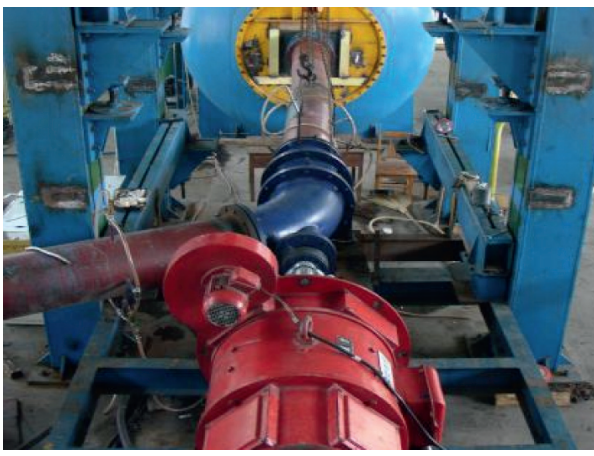

(c)

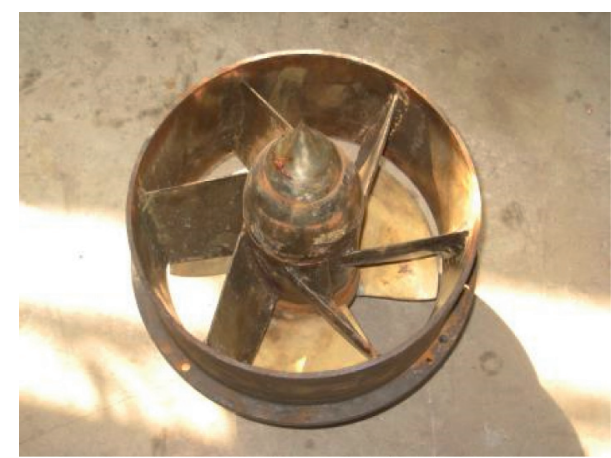

(b)

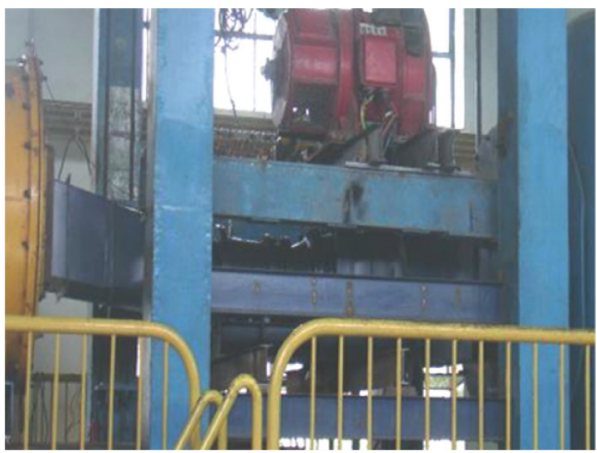

(d)

FIgURe 2: Pictures of experiment: (a) impeller; (b) guide vane; (c) physical diagram of test equipment of the axial-flow pump; (d) physical diagram of test equipment of the axial-flow pump device.

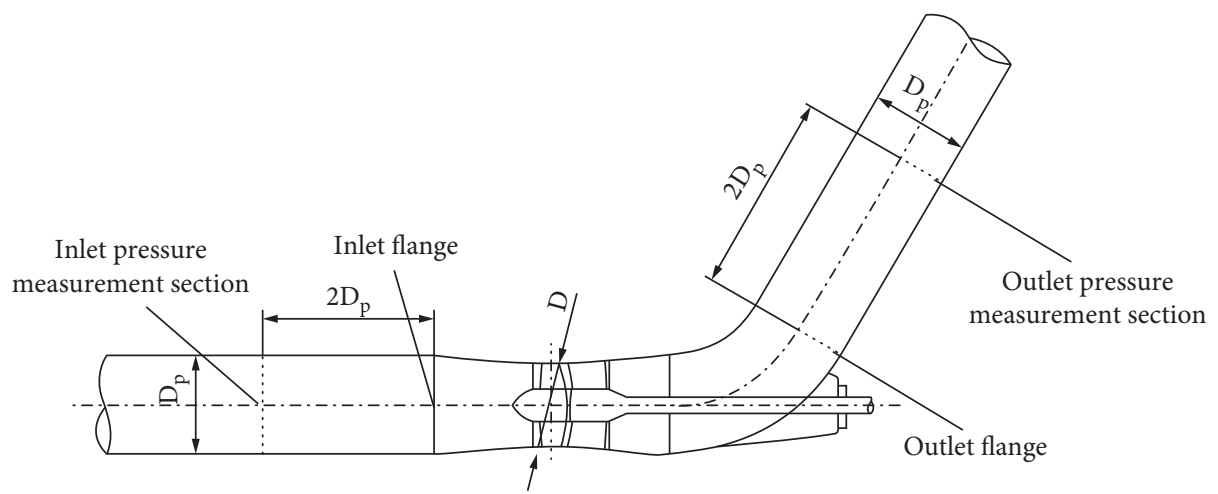

(a)

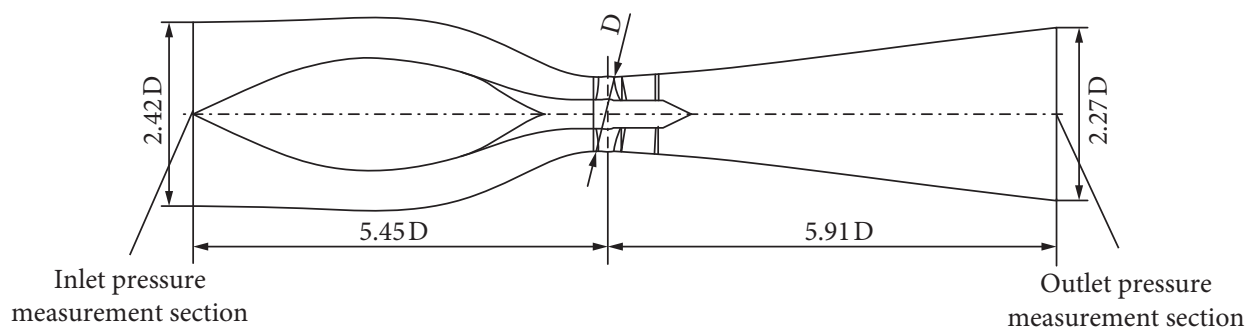

(b)

FIgURE 3: Location of pressure measurement section: (a) axial-flow pump; (b) axial-flow pump device.

hydraulic test bench is composed of a hydraulic circulation device, power control device, and data acquisition and computer analysis device. The tests were completed under the requirements of Chinese standards. The information about the test instruments is shown in Table 1. The tests were completed under the requirements of 
TABLE 1: Main test instruments of test bench.

\begin{tabular}{lccc}
\hline Measuring items & Instrument & Instrument model & Accuracy $(\%)$ \\
\hline Head & Differential pressure transmitter & LDG-500s & \pm 0.1 \\
Flow rate & Electromagnetic flowmeter & V15712-HD1A1D7D & \pm 0.2 \\
Torque and rotation speed & Torque and speed sensor & JCZL2-500 & \pm 0.1 \\
\hline
\end{tabular}

Chinese standards. The performance of the axial-flow pump and pump device at different blade mounting angles of $-4^{\circ},-2^{\circ}, 0^{\circ}$, and $+2^{\circ}$ was tested. The performance curves at different blade mounting angles were obtained from more than 15 test points.

The uncertainty of the head measurement system is less than $\pm 0.1 \%$, the uncertainty of the torque and rotation speed measurement system is less than $\pm 0.1 \%$, and the uncertainty of the flow rate measurement system is less than $\pm 0.2 \%$; according to the above uncertainty, the uncertainty of the test bench system is calculated to be $\pm 0.25 \%$. In this study, the data of the axial-flow pump device with blade angle of $-4^{\circ}$ under optimal conditions were collected 10 times, and the efficiency of each time was calculated, and then the average efficiency and standard deviation were calculated. Student's $t$-distribution is adopted with a confidence of $95 \%$, and the random uncertainty of the efficiency test is $\pm 0.0728 \%$. According to the system uncertainty and random uncertainty, the comprehensive uncertainty of the efficiency test is $\pm 0.26 \%$. Table 2 shows the efficiency data of 10 repetitions.

Figure 4 shows the model test results. The performance curves at different angles exhibit basically the same trend. Figure 5 compares the $Q-H$ curves at different blade mounting angles. It can be found that there are two saddleshaped regions of the pump, while there is only one saddleshaped region of the corresponding pump device, and this is consistent with the results in $[12,13]$. For the convenience of discussion, the saddle-shaped region with larger flow rate is called the first saddle-shaped region of the axial-flow pump, and the other with the smaller flow rate is called the second saddle-shaped region. The lowest head of the first saddleshaped region is called the first saddle bottom head. The lowest head of the second saddle-shaped region is called the second saddle bottom head. The saddle bottom head of the pump and its corresponding pump device at different angles was statistically analyzed and is shown in Tables 3 and 4 . It can be found that at different blade angles, the first saddle bottom head of the pump is close to the saddle bottom head of the corresponding pump device, while the second saddle bottom head is obviously lower than the saddle bottom head of the corresponding pump device.

As the trend of performance curves at different blade mounting angles is basically the same, in order to describe in detail, two curves with blade mounting angle of $0^{\circ}$ were compared, and the $x$ axis was replaced by the ratio of flow rate to design flow rate $\left(Q_{d}\right)$. The comparison is shown in Figure 6. Under the design condition, the efficiency of pump measured by the model test is $85.74 \%$, and the head measured by the model test is $5.20 \mathrm{~m}$. When the axial-flow pump is operating at $0.6 \sim 1.2 Q_{d}$, the head increases with decreasing flow rate, and the head exhibits one-to-one correspondence with the flow rate, so this region is called the stable operating region. When the flow rate is less than $0.6 Q_{d}$, the curve fluctuates, forming two saddle-shaped regions. In this region, one head corresponds to multiple flow rate values, and thus this region is called the unstable operating region of the pump.

According to Figure 6, under the design condition, the efficiency of the pump device measured by the model test is $81.77 \%$, and the model test head is $4.82 \mathrm{~m}$. The head of the pump device head is $0.38 \mathrm{~m}$ lower than that of the pump, and the efficiency of the pump device is $3.97 \%$ lower than that of the pump. This is because under the design condition, the hydraulic loss of inlet and outlet passage is greater than that of inlet and outlet pipe, so the head and efficiency of the pump device will be lower. When the axial-flow pump device is operating at $0.6 \sim 1.2 Q_{d}$, the head increases with decreasing flow rate, and the curve exhibits a quadratic relationship. In this operation region, the head exhibits one-to-one correspondence with the flow rate, so this region is called the stable operation region. When the flow rate is less than $0.6 Q_{d}$, the head decreases in the range of $0.45 \sim 0.6 Q_{d}$ and then increases. In this region, the curve fluctuates, forming one saddle-shaped region, and the head corresponds to multiple flow rate values. Thus, this region is called the unstable operating region of the pump device. Comparing the $Q-H$ curves of the pump and corresponding pump device, it is found that the two curves are basically the same in the stable operation region. In the unstable operation region, it is found that there are two saddle-shaped regions of the axial-flow pump, while there is only one saddle-shaped region of corresponding pump device.

\section{Numerical Calculations}

3.1. Computational Model. To research the difference of saddle-shaped region of $Q-H$ curves, numerical calculations of the axial-flow pump with a blade angle of $0^{\circ}$ and the axialflow pump device with a blade angle of $0^{\circ}$ were completed. The size of the calculation domain is the same as the model test, and the arrangement of pressure measurement points is also consistent with the model test. Figures 7 and 8 show the three-dimensional models of each component in the calculation domain.

3.2. Turbulence Model and Boundary Conditions. In recent years, the numerical simulation method has become a common method for the study of rotating machinery, such as the axial-flow pump [18-22], centrifugal pump [23-26], multistage multiphase pump [27-29], pump as turbine [30], and so on. In this numerical simulation, the time-averaged Navier-Stokes equation is adopted to describe flow [31, 32]. 
TABle 2: Efficiency data.

\begin{tabular}{lcccccccccc}
\hline Number & 1 & 2 & 3 & 4 & 5 & 6 & 7 & 8 & 9 & 10 \\
\hline Efficiency (\%) & 82.39 & 82.19 & 82.28 & 82.33 & 82.36 & 82.38 & 82.21 & 82.17 & 82.21 & 82.27 \\
\hline
\end{tabular}

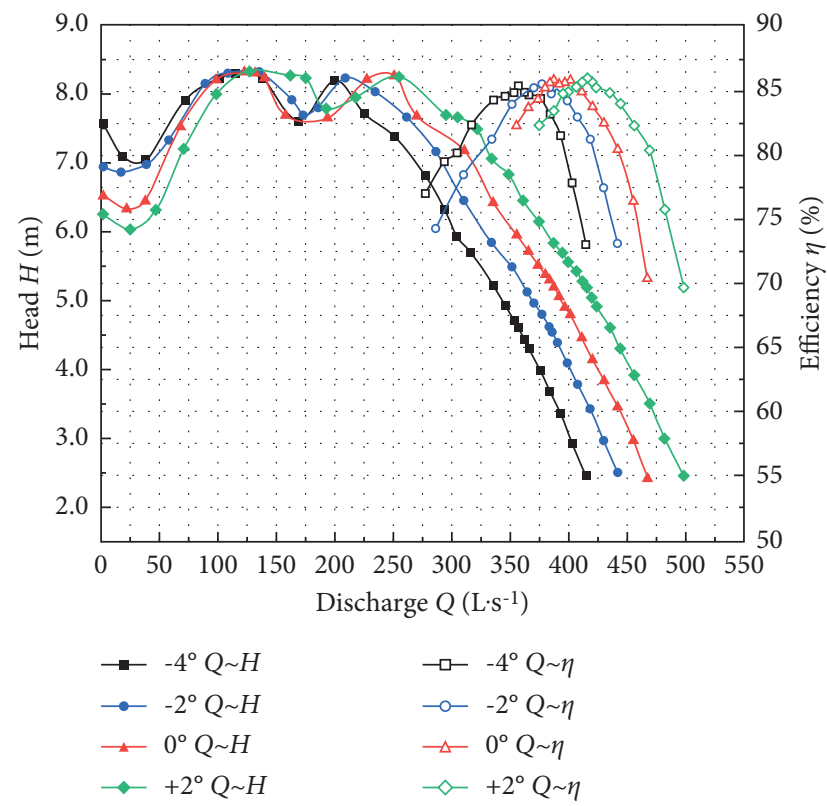

(a)

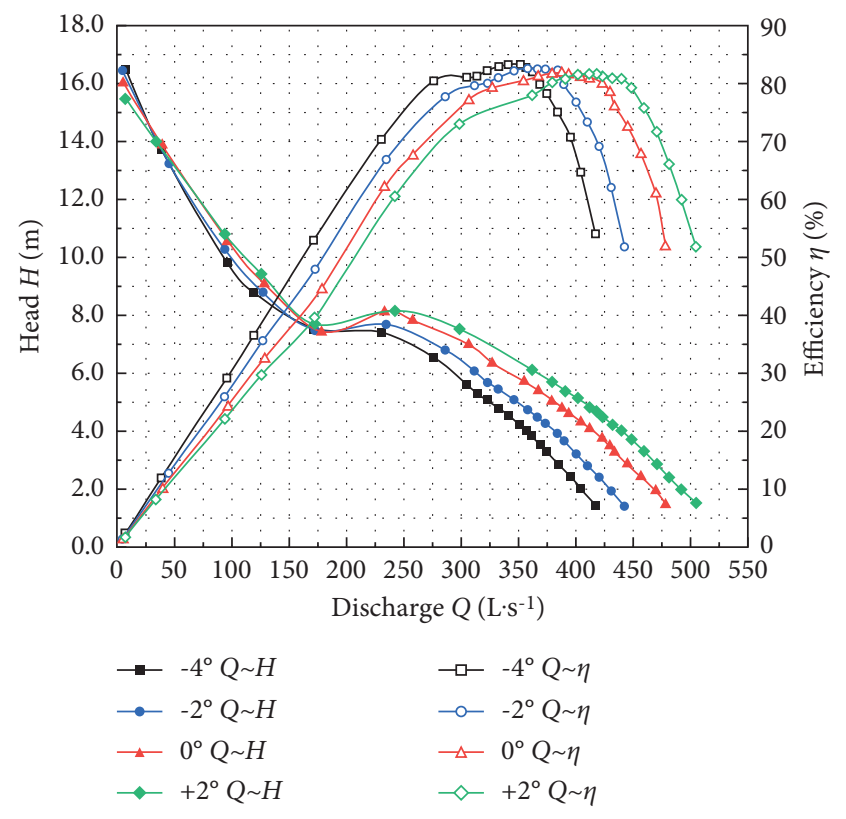

(b)

Figure 4: Performance curve: (a) axial-flow pump; (b) axial-flow pump device.

Because of the advantage of the RNG $k$ - $\varepsilon$ turbulence model in solving the flow field of rotating machinery, the model is used in this paper [33-37]. In this study, the inlet boundary condition was a standard atmospheric pressure, and the outlet was set as the mass flow outlet and all solid surfaces were set to wall. Water temperature is $25^{\circ} \mathrm{C}$, regardless of heat transfer. The equations are shown below:

$$
\begin{aligned}
& \frac{\partial}{\partial t}(\rho k)+\frac{\partial}{\partial x_{i}}\left(\rho k u_{i}\right)=\frac{\partial}{\partial x_{j}}\left(\alpha_{k} \mu_{\mathrm{eff}} \frac{\partial k}{\partial x_{j}}\right)+G_{k}+\rho \varepsilon, \\
& \frac{\partial}{\partial t}(\rho \varepsilon)+\frac{\partial}{\partial x_{i}}\left(\rho \varepsilon u_{i}\right)=\frac{\partial}{\partial x_{j}}\left(\alpha_{\varepsilon} \mu_{\mathrm{eff}} \frac{\partial \varepsilon}{\partial x_{j}}\right)+\frac{C_{1 \varepsilon} \varepsilon}{k} G_{k}-C_{2 \varepsilon} \rho \frac{\varepsilon^{2}}{k}
\end{aligned}
$$

where $G_{k}$ represents the turbulent kinetic energy generated by the average velocity gradient; $C_{1 \varepsilon}=1.42$ and $C_{2 \varepsilon}=1.68 ; \mu_{\text {eff }}$ is the effective viscosity of turbulence; $k$ is the turbulence energy; and $\varepsilon$ is the dissipation rate of the turbulent kinetic energy.

3.3. Grid Division and Irrelevance Verification. According to the design parameters, inlet pipe, shaft inlet passage, waterguide cone, outlet pipe, and straight outlet passage are modeled, and the hexahedral structured grid is divided in ICEM software. The impeller and guide vane are modeled according to coordinate points and divided into structural grids in TurboGrid software. A large number of research studies show that the value of $y^{+}$and the setting of tip clearance have a great influence on the calculation accuracy [38-40]. The tip clearance was $0.2 \mathrm{~mm}$ in this calculation. The $y^{+}$value of the impeller wall was 18 . In this study, six sets of grids were divided for the calculation domain, and the efficiency under design condition was used as an index to judge grid independence. Figure 9 shows the verification results. The grid division is shown in Figure 10.

Figure 9(a) shows that the efficiency of the pump is almost constant when the grid number is more than 4.92 million. Figure 9(b) shows that the efficiency of the pump device is almost constant when the grid number is more than 6.29 million. After comprehensive consideration of calculation accuracy and computing resources, 4.92 million and 6.29 million were finally selected.

\section{Results and Discussion}

4.1. Numerical Simulation Results. To verify the accuracy of simulation, the experimental results were compared with the numerical simulation results. In this study, the head was calculated according to the Bernoulli equation. The efficiency was predicted according to the velocity, pressure, and torque on the impeller. Comparison between numerical and experimental results is shown in Figure 11.

The calculation formula of the head is expressed as follows: 


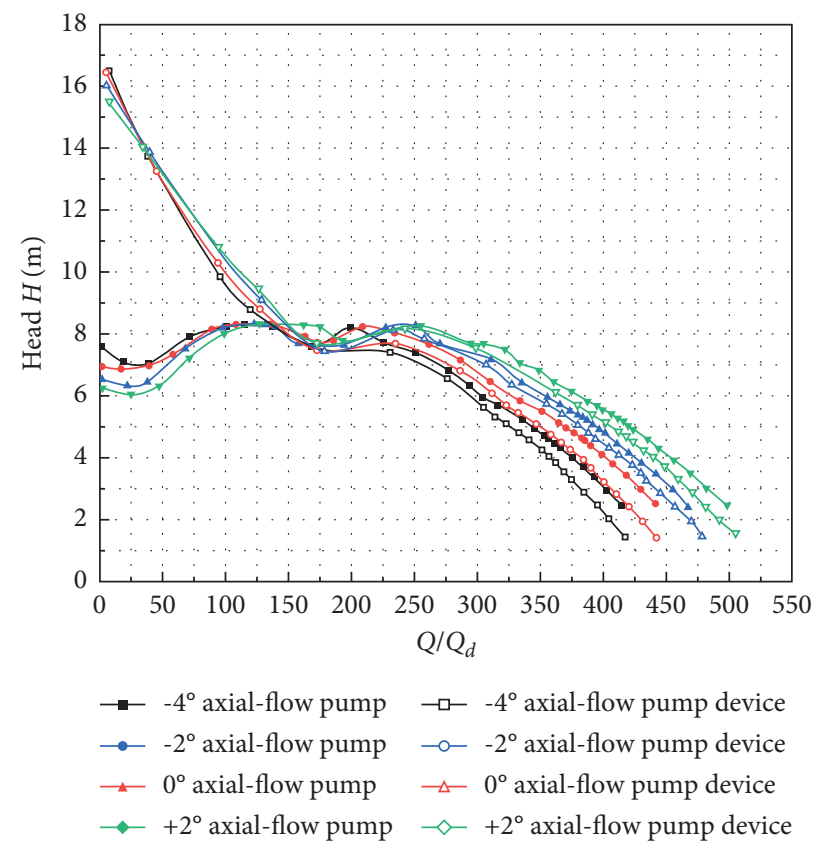

Figure 5: The comparison of $Q-H$ curves at different blade angles.

TABLE 3: Saddle bottom head of the axial-flow pump.

\begin{tabular}{lccrr}
\hline Blade mounting angle $\left(^{\circ}\right)$ & -4 & -2 & 0 & +2 \\
\hline The first saddle bottom head $(\mathrm{m})$ & 7.64 & 7.74 & 7.62 & 7.78 \\
The second saddle bottom head $(\mathrm{m})$ & 7.06 & 6.88 & 6.21 & 6.00 \\
\hline
\end{tabular}

TABLE 4: Saddle bottom head of the axial-flow pump device.

\begin{tabular}{lcccc}
\hline Blade mounting angle $\left(^{\circ}\right)$ & -4 & -2 & 0 & +2 \\
\hline First saddle bottom head $(\mathrm{m})$ & 7.50 & 7.63 & 7.50 & 7.68 \\
\hline
\end{tabular}

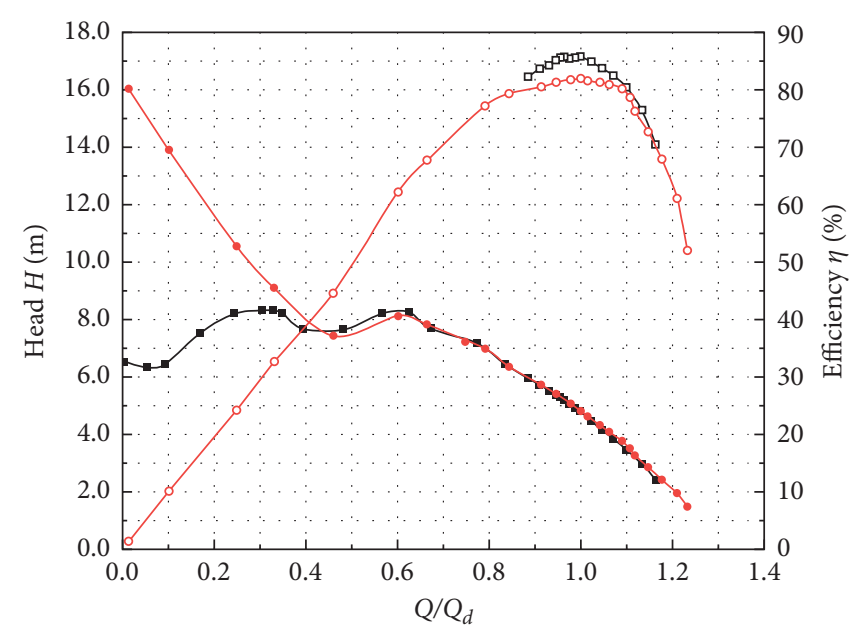

$$
\begin{aligned}
& \rightarrow \text { test of axial flow-pump } Q \sim H \\
& \rightarrow \text { test of axial flow-pump device } Q \sim H \\
& \rightarrow-\text { test of axial flow-pump } Q \sim \eta \\
& \multimap-\text { test of axial flow-pump device } Q \sim \eta
\end{aligned}
$$

Figure 6: The performance comparison between the axial-flow pump and axial-flow pump device. 


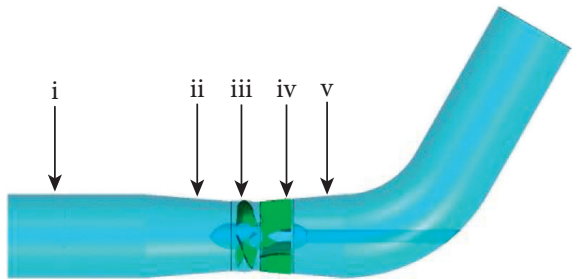

FIgURE 7: Computational domain of the axial-flow pump: (i) inlet pipe; (ii) inlet cone pipe; (iii) impeller; (iv) guide vane; (v) outlet pipe.

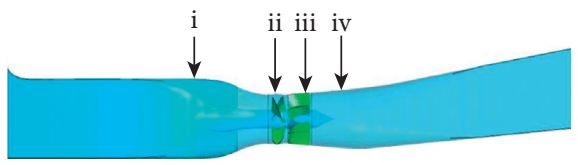

FIGURE 8: Computational domain of the axial-flow pump device: (i) inlet passage; (ii) impeller; (iii) guide vane; (iv) outlet passage.

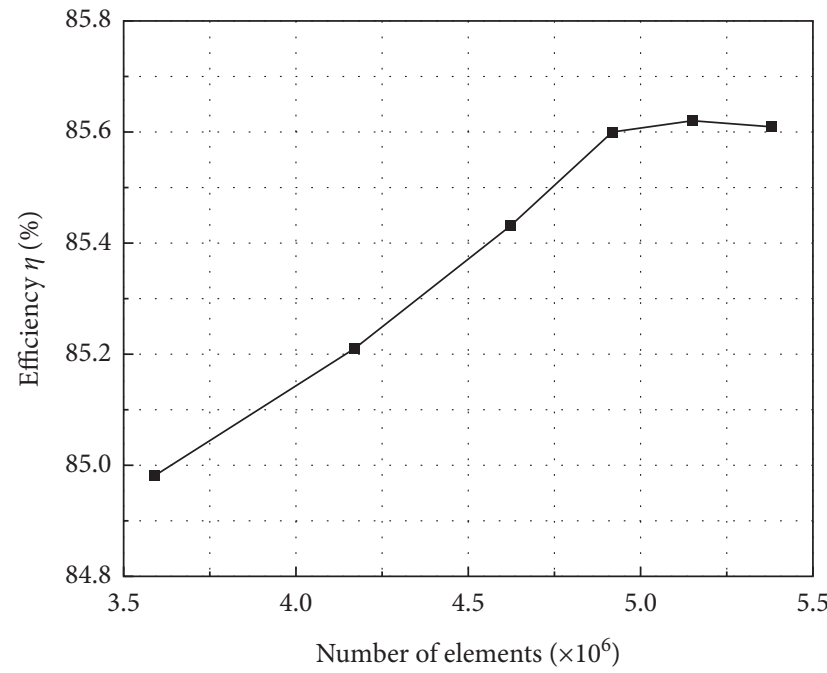

(a)

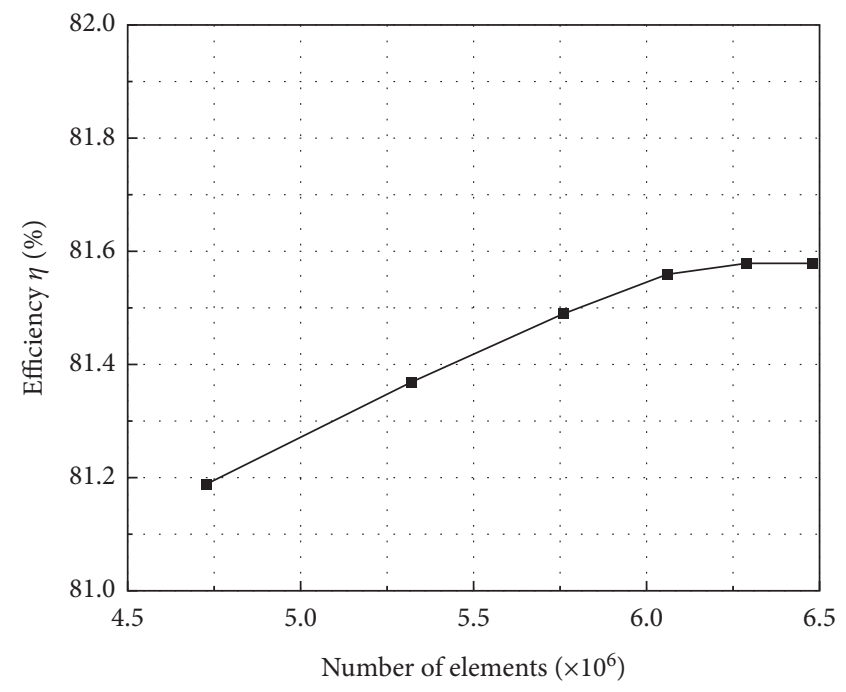

(b)

FIGURE 9: Grid independence analysis: (a) axial-flow pump; (b) axial-flow pump device.

$$
H=\frac{\left(P_{\text {out }}^{T}-P_{\text {in }}^{T}\right)}{\rho g},
$$

where $P_{\text {out }}^{T}$ and $P_{\text {in }}^{T}$ represent the total pressure of outlet and inlet pressure measurement section, respectively, $\mathrm{Pa}$.

The calculation formula of hydraulic efficiency is expressed as follows:

$$
\eta=\frac{\rho g Q H}{T_{p} \omega},
$$

where $T_{p}$ is torque, $\mathrm{N} \cdot \mathrm{m}$.

According to Figure 11(a), in the design condition, the experimental efficiency value is $85.74 \%$, the efficiency predicted by the CFD is $85.62 \%$, the absolute error of efficiency is $0.12 \%$, and the relative error of efficiency is $0.14 \%$. The experimental head value is $5.20 \mathrm{~m}$, the head predicted by the CFD is $5.05 \mathrm{~m}$, the absolute error of the head is $0.15 \mathrm{~m}$, and the relative error of the head is $2.9 \%$. According to Figure 11(b), in the design condition, the experimental efficiency value is
$81.77 \%$, the efficiency predicted by the CFD is $81.58 \%$, the absolute error of efficiency is $0.19 \%$, and the relative error of efficiency is $0.23 \%$. The experimental head value is $4.82 \mathrm{~m}$, the head predicted by the CFD is $4.61 \mathrm{~m}$, the absolute error of the head is $0.21 \mathrm{~m}$, and the relative error of the head is $4.3 \%$. The error between numerical simulation and test is very small in both design and non-design conditions, indicating that the CFD method is reliable.

4.2. Internal Flow of the Impeller. This study established different blade spans; span represents the blade span, span=0 represents the hub position, and span=1 represents the rim positioned. Figure 12 shows the internal flow characteristics for the blade spans under different working conditions. According to Figure 12, when the flow rate is $1.0 Q_{d} \sim 0.8 Q_{d}$, the flow pattern is smooth and ordered, the flow moves along the blade airfoil, and there is no backflow in the impeller. When the flow rate is $0.6 Q_{d}$, there is a backflow zone near the impeller rim, but the backflow zone is small, whereas swirl 


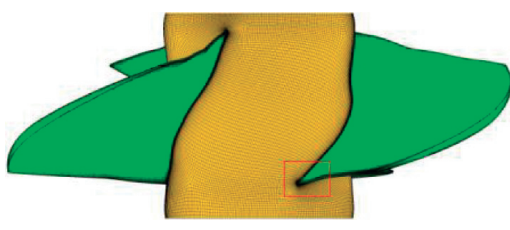

(a)

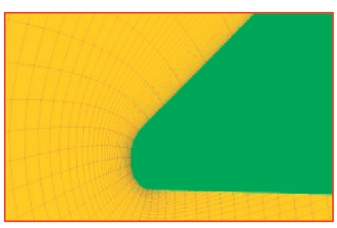

(b)

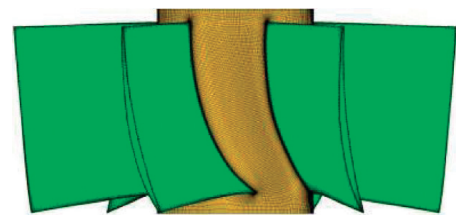

(c)

Figure 10: Figures of grids: (a) impeller; (b) grid refinement of impeller tip; (c) guide vane.

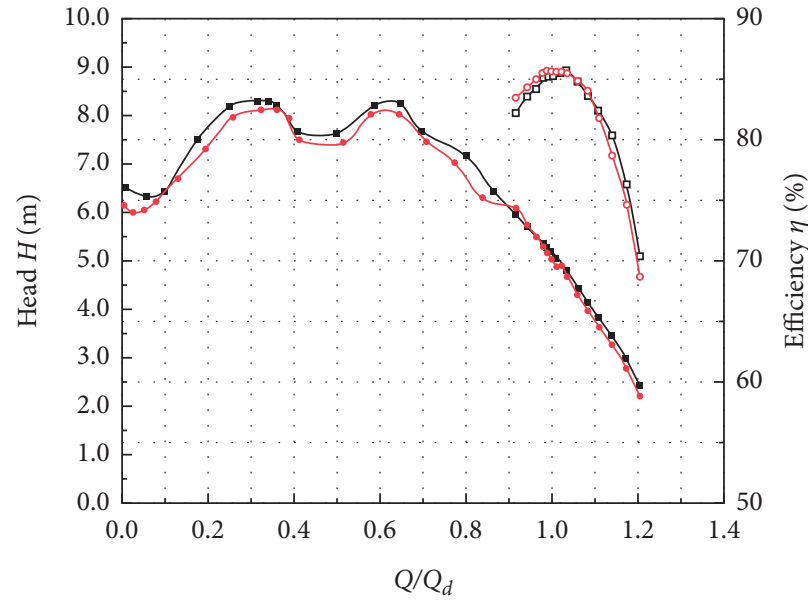

$$
\begin{aligned}
& - \text { test of axial flow pump Q-H } \\
& \rightarrow \text { CFD of axial flow pump Q-H } \\
& \rightarrow \text { test of axial flow pump Q- } \eta \\
& \multimap \text { CFD of axial flow pump } Q-\eta
\end{aligned}
$$

(a)

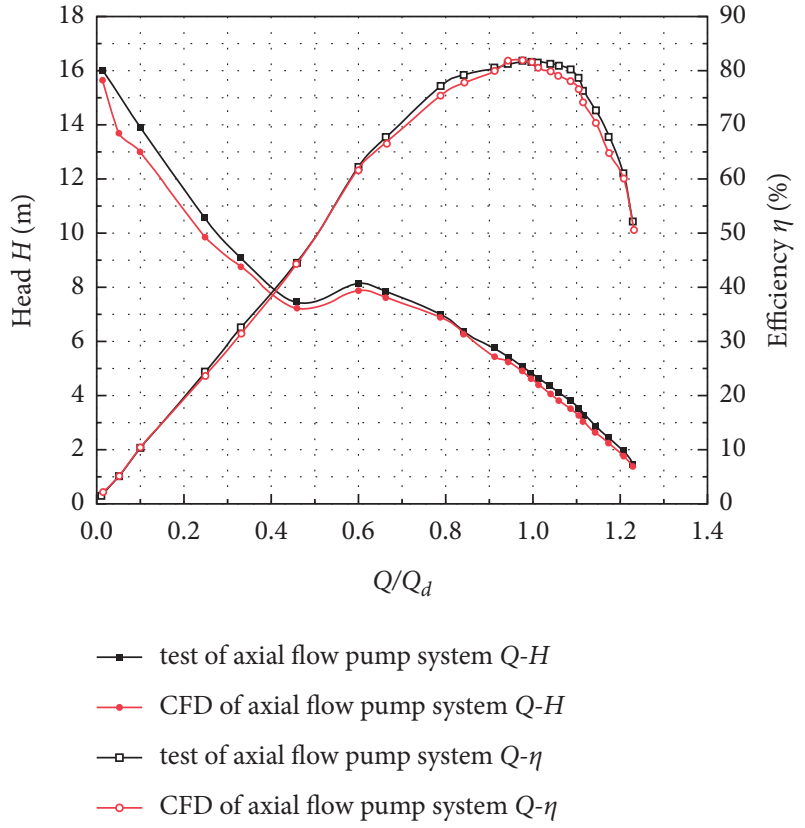

(b)

FIgURE 11: Comparison of the experimental and numerical simulation results: (a) axial-flow pump; (b) axial-flow pump device.

appears near the impeller hub. When the flow is $0.32 Q_{d}$, the flow pattern is disordered, and the backflow zone near the impeller hub and rim increases greatly. When the flow rate continues to decrease to $0.12 Q_{d}$, the backflow zone in the impeller further increases, whereas the backflow zone near the impeller rim occupies almost the whole back of the blade. It was also found in reference [16] that there was backflow at the inlet of the impeller under the condition of small flow rate.

According to Figures 11 and 12, when the flow rate is about $0.6 Q_{d}$, the head begins to decrease, the flow pattern of the impeller is disordered, and backflow phenomenon is observed. This is due to the impact angle of water flow and blade increasing with the decrease of flow rate. When the impact angle increases to a certain extent, the lift coefficient decreases. Therefore, the first saddle-shaped region of the axial-flow pump and the saddle-shaped region of the corresponding pump device are the external characteristic responses of the pump's internal flow characteristics.

\subsection{Internal Flow of Inlet Pipe and Shaft Inlet Passage.} The backflow at the inlet section of the impeller enters the inlet straight pipe or the inlet passage, thus affecting the internal flow in the inlet pipe or the inlet passage. For further study, the internal flow of inlet pipe and shaft inlet passage are removed for comparison. Figure 13 shows the internal flow of inlet pipe. Figure 14 shows the axial velocity distribution of the inlet pressure measurement section of the axial-flow pump test. Figure 15 shows the internal flow of shaft inlet passage.

According to Figures 13 and 14, when the axial-flow pump is operating at $0.8 \sim 1.0 Q_{d}$, the flow in the inlet pipe is uniform and smooth without backflow. When the flow decreases to $0.6 Q_{d}$, the backflow occurs at the impeller inlet near the rim, but it is small. When the flow rate is $0.32 Q_{d}$, the backflow zone in the inlet pipe increases greatly along the radial and axial directions. The axial velocity distribution of the inlet pressure measurement section is uniform, and the axial velocity value is basically the same when the flow rate is greater than $0.6 Q_{d}$. However, due to the influence of backflow, the axial velocity in the inner and outer sides of the section is inconsistent, and the axial velocity decreases from the center to the outer, which may lead to inconsistent pressure distribution in the section when the flow rate is less than $0.32 Q_{d}$. Zheng et al. [15] also found the same backflow phenomenon but did not determine the influence range of 


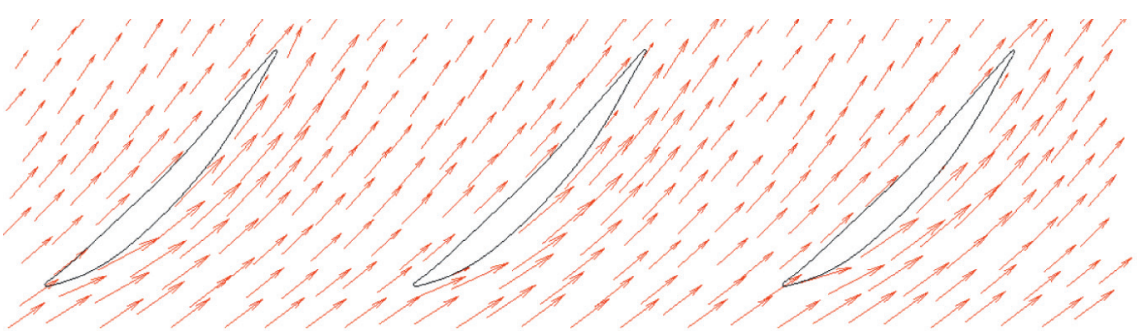

Span $=0.1$
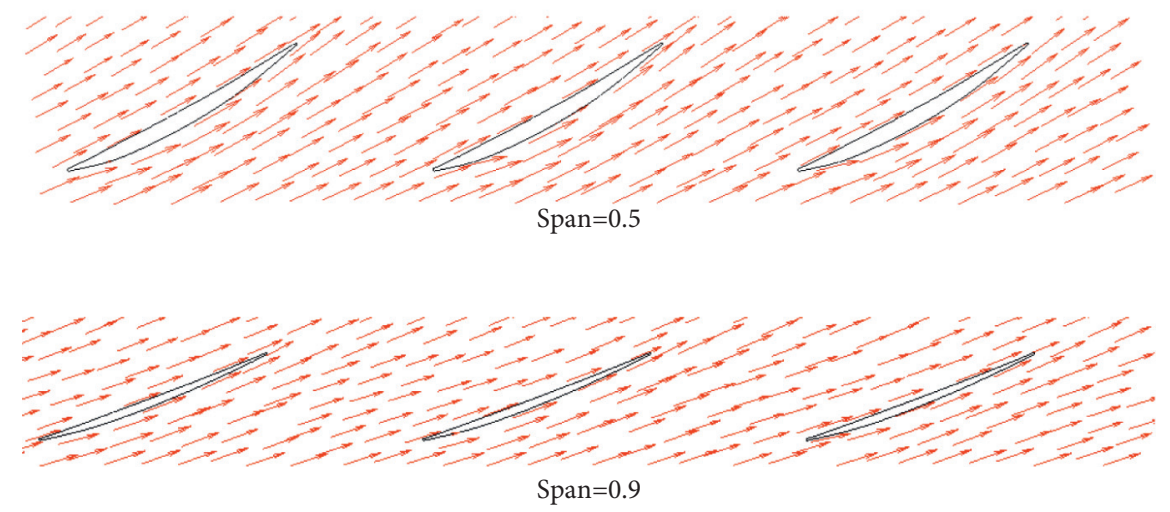

(a)
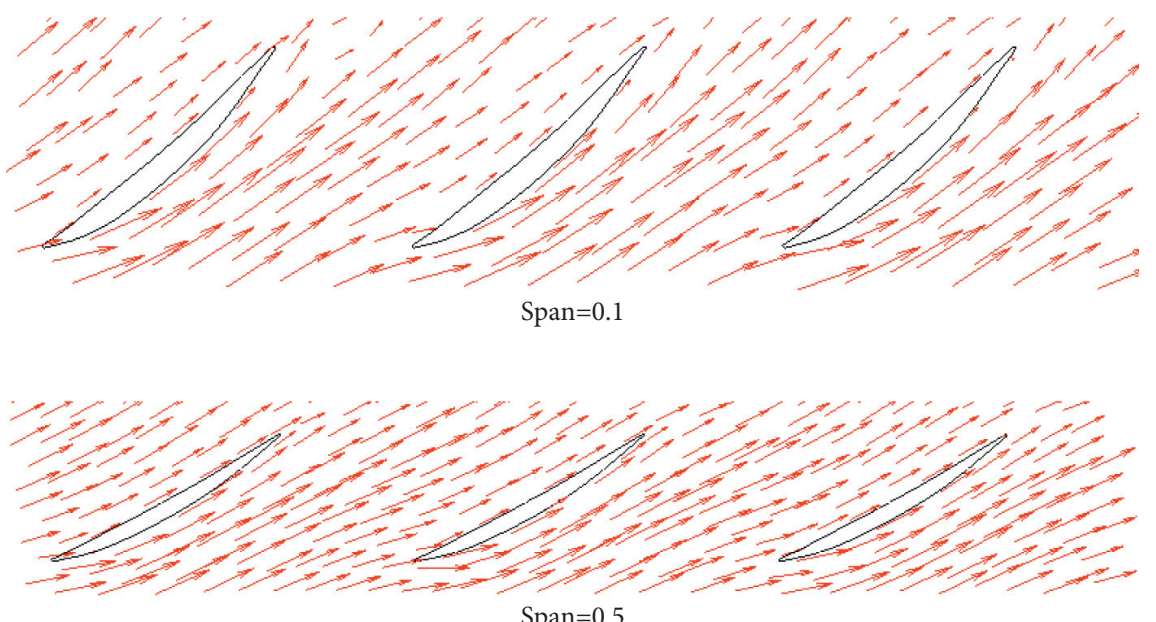

Span $=0.5$

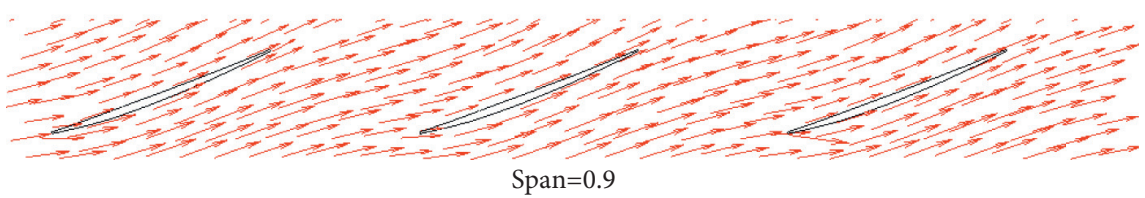

(b)

FIgURE 12: Continued. 

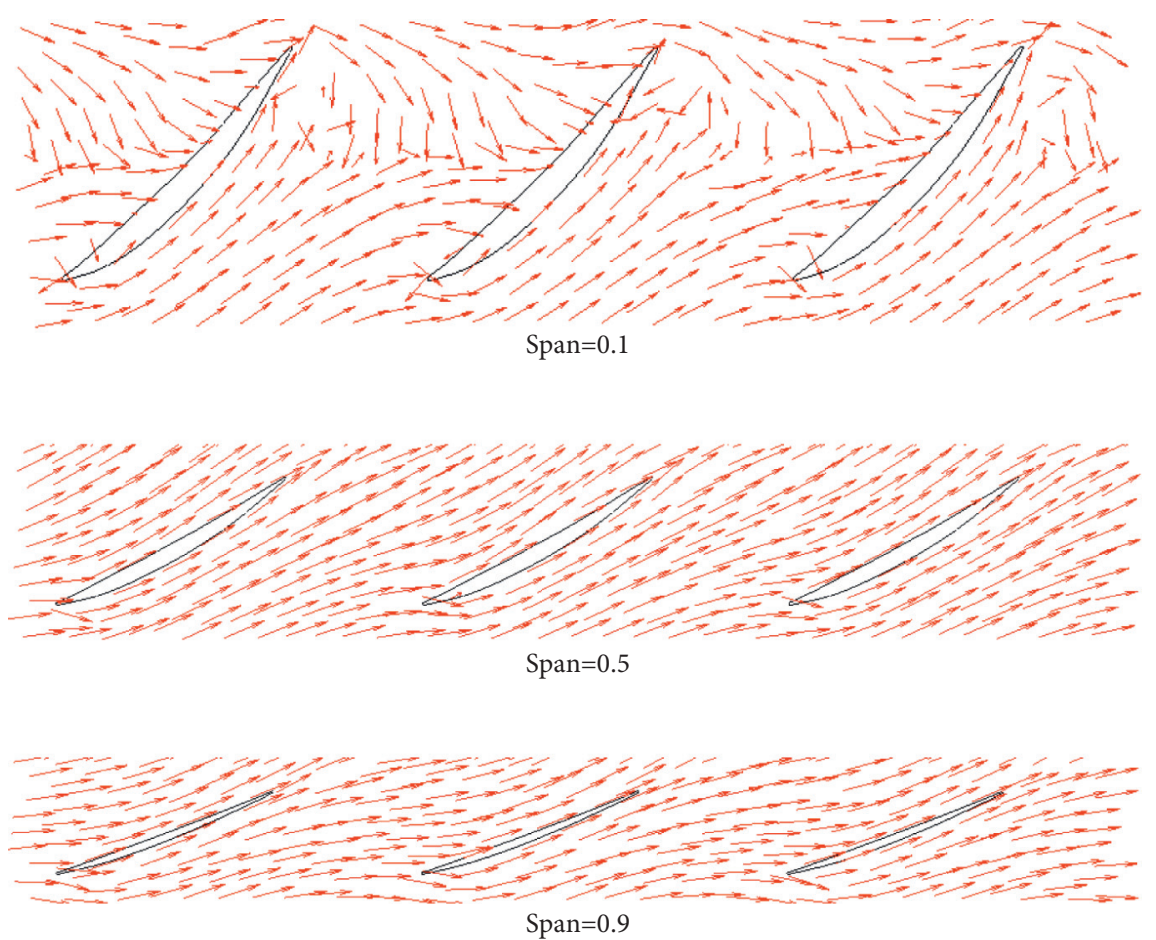

(c)
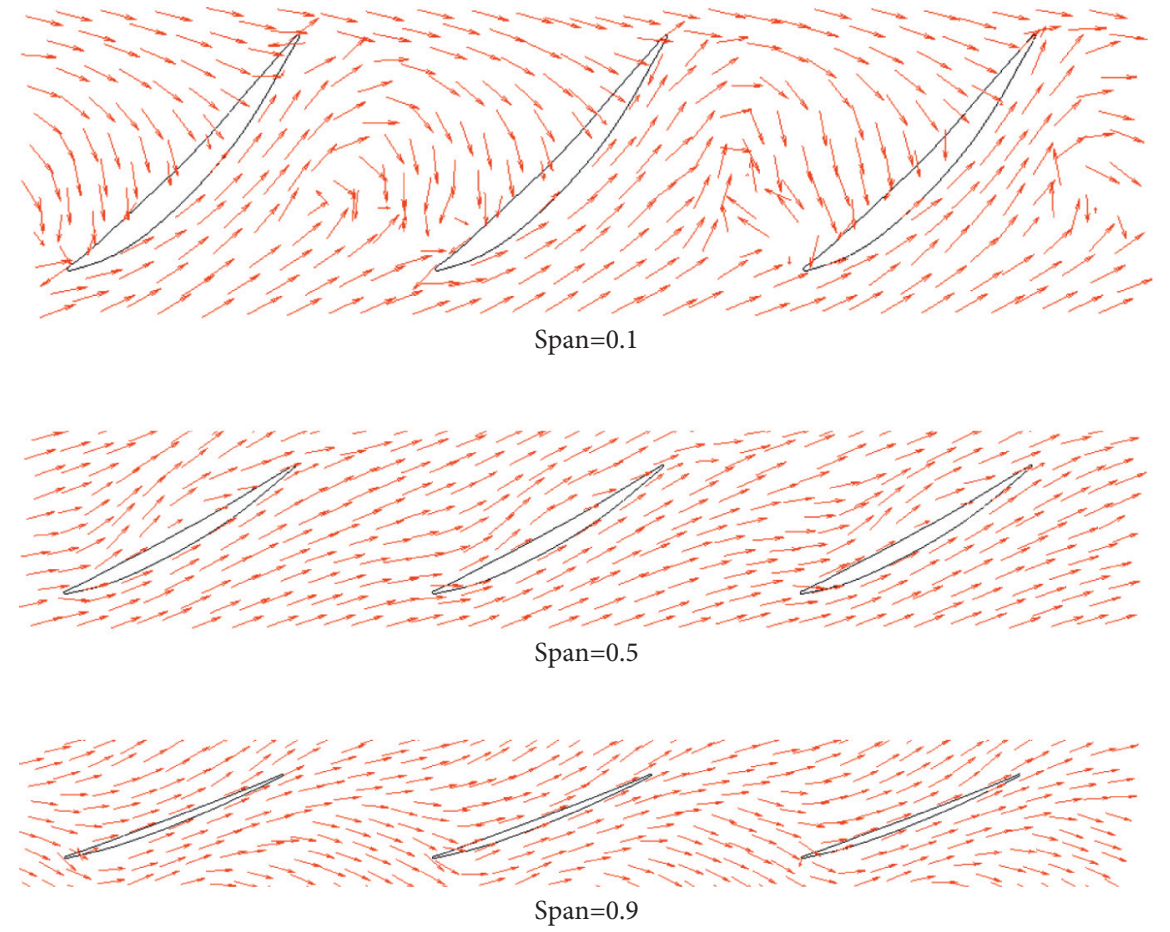

(d)

Figure 12: Continued. 

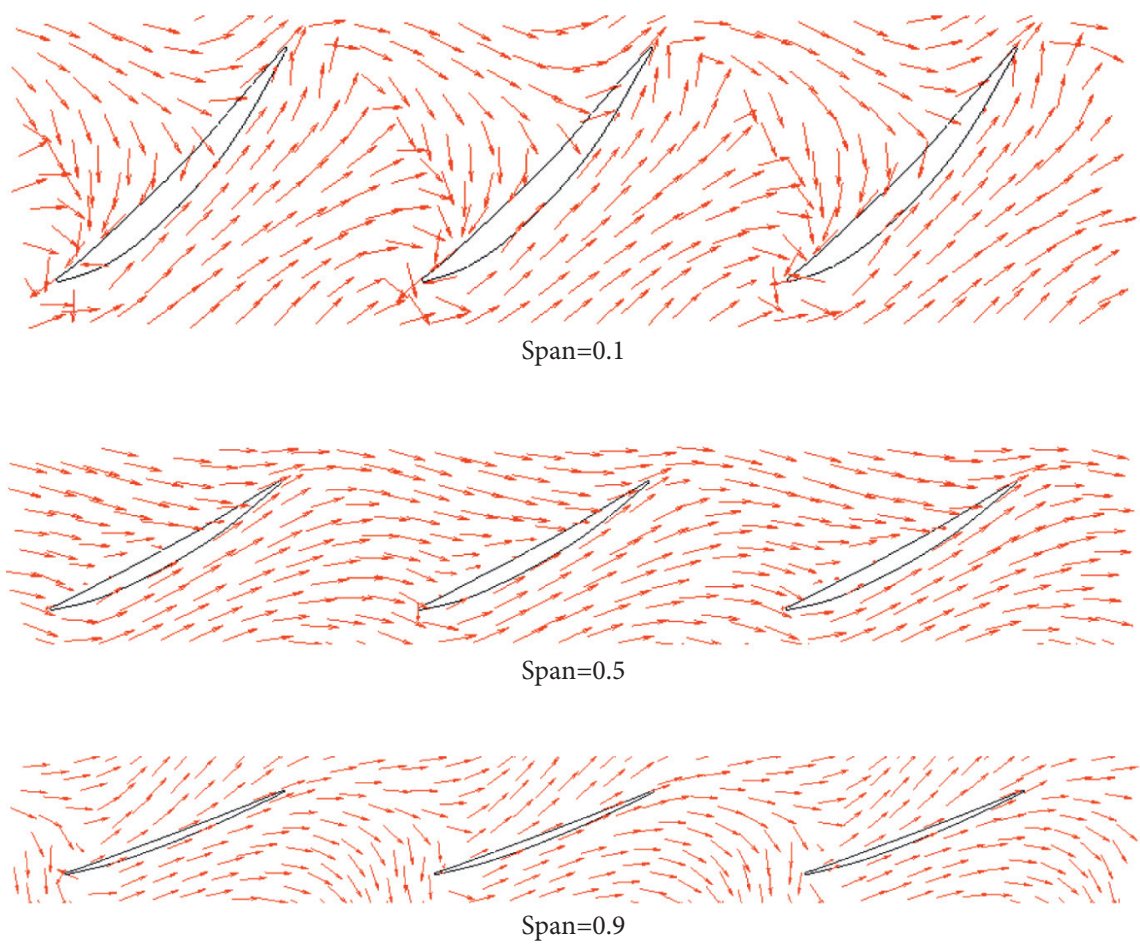

(e)

Figure 12: Flow pattern of the impeller: (a) $Q / Q_{d}=1.0$; (b) $Q / Q_{d}=0.80$; (c) $Q / Q_{d}=0.60$; (d) $Q / Q_{d}=0.32$; (e) $Q / Q_{d}=0.12$.

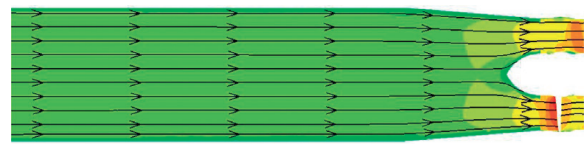

Axial Velocity (m/s)

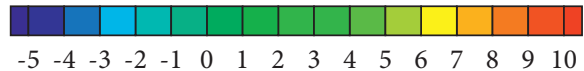

(a)

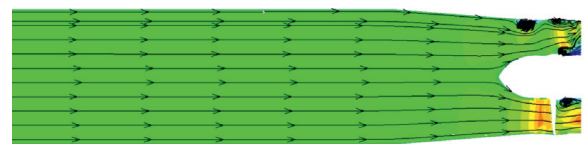

Axial Velocity (m/s)

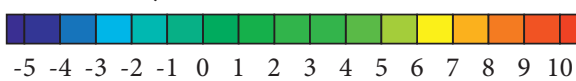

(c)

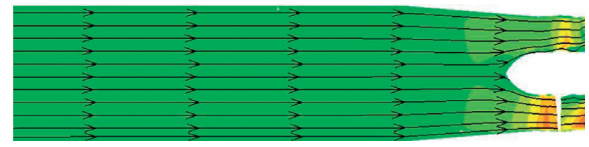

Axial Velocity (m/s)

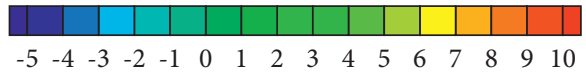

(b)

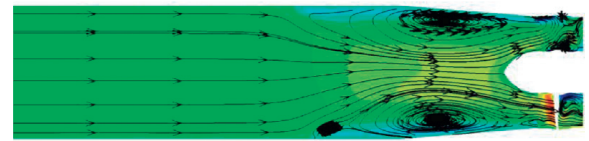

Axial Velocity $(\mathrm{m} / \mathrm{s})$

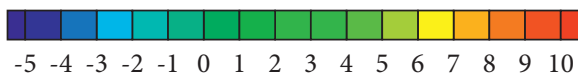

(d)

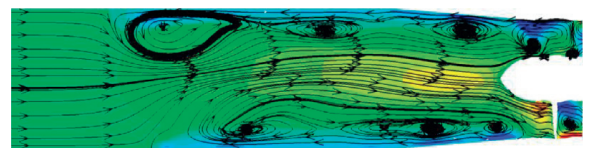

Axial Velocity (m/s)

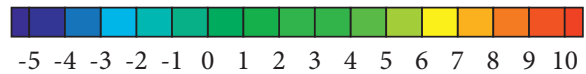

(e)

FIGURE 13: Internal flow pattern of the inlet pipe under different conditions: (a) $Q / Q_{d}=1.0$; (b) $Q / Q_{d}=0.8$; (c) $Q / Q_{d}=0.6$; (d) $Q / Q_{d}=0.32$; (e) $Q / Q_{d}=0.12$. 


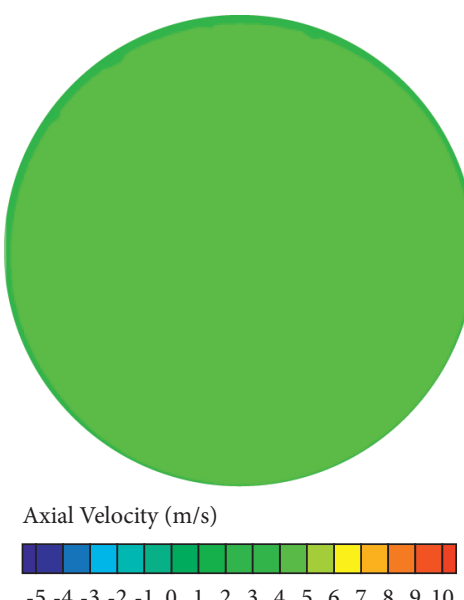

(a)

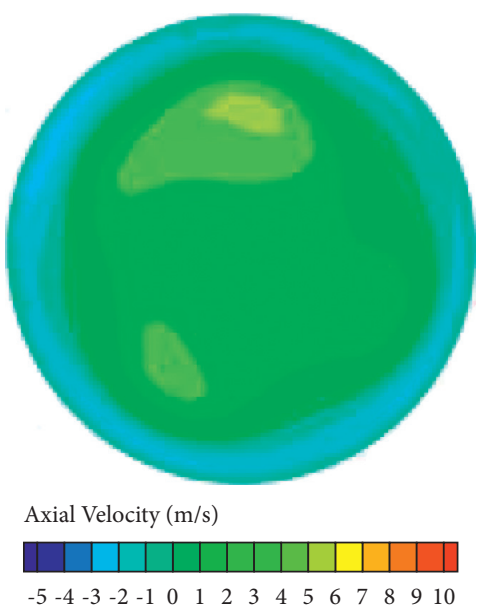

(d)

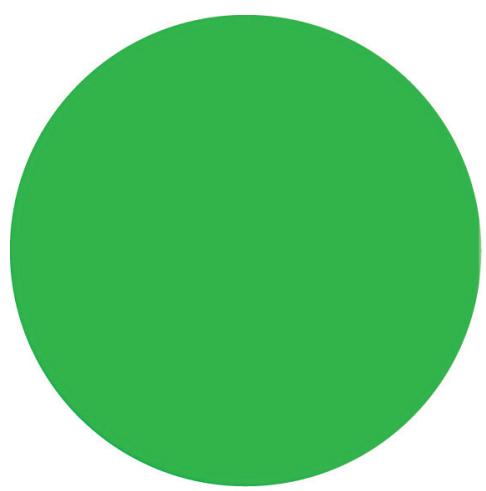

Axial Velocity (m/s)

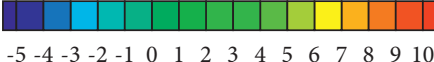

(b)

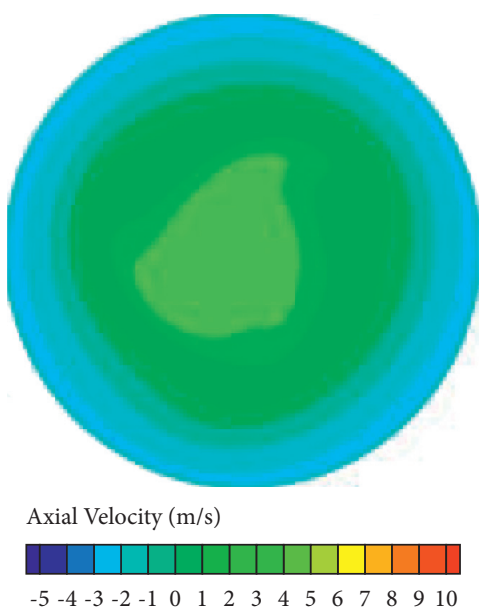

(e)

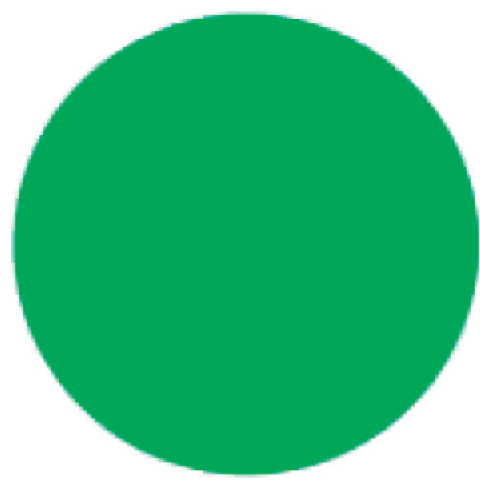

Axial Velocity (m/s)

$\begin{array}{llllllllllllllll}-5 & -4 & -3 & -2 & -1 & 0 & 1 & 2 & 3 & 4 & 5 & 6 & 7 & 8 & 9 & 10\end{array}$

(c)

FIGURE 14: Axial velocity distribution of the inlet pressure measurement section of the axial-flow pump: (a) $Q / Q_{d}=1.0$; (b) $Q / Q_{d}=0.8$; (c) $Q /$ $Q_{d}=0.6$; (d) $Q / Q_{d}=0.32$; (e) $Q / Q_{d}=0.12$.

the backflow in combination with the position of the inlet pressure measurement section.

According to Figure 15, When the axial-flow pump device is operating at $0.8 \sim 1.0 Q_{d}$, the flow in the inlet passage is uniform and smooth without backflow. When the flow decreases to $0.6 Q_{d}$, the backflow phenomenon is observed. With the decrease of flow rate, the range of backflow zone increases along the axial and radial directions. Since the inlet pressure measurement section is set far away from the inlet of the impeller, it will not be affected by the backflow in all working conditions.

4.4. Internal Pressure Distribution. According to the above analysis, for the axial-flow pump test, the flow field of the inlet pressure measurement section will be affected by the backflow at low flow rate, resulting in an uneven axial velocity distribution in the section. The pressure distribution of inlet pressure measurement section under different working conditions was compared. For further study, the pressure distribution of different inlet pressure measuring sections was compared and the head was calculated according to the pressure of different sections. The position of inlet pressure measurement section $\mathrm{A}$ is the position of the inlet pressure measurement section in the axial-flow pump model test, and the inlet pressure measurement section $\mathrm{B}$ is $4 D_{p}$ from the inlet flange of the impeller chamber. The location of different sections is shown in Figure 16. The pressure distributions of different sections are shown in Figures 17 and 18, respectively. Different $Q-H$ curves are shown in Figure 19.

When the flow rate is $1.0 Q_{d} \sim 0.6 Q_{d}$, the pressure distribution in inlet pressure measurement section $\mathrm{A}$ is uniform, and the pressure near the outside and inside of the inlet pipe is almost the same. When the flow is lower than $0.32 Q_{d}$, the pressure is not uniform, and a high-pressure zone is formed in the rim of inlet pipe. With the decrease of the flow rate, the pressure in the high-pressure zone becomes 


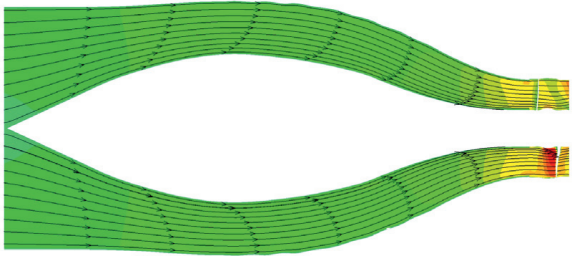

Axial Velocity $(\mathrm{m} / \mathrm{s})$

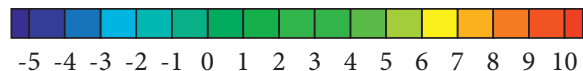

(a)

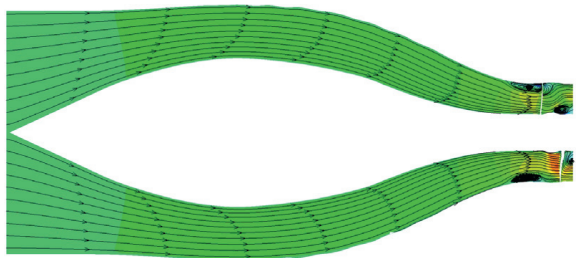

Axial Velocity $(\mathrm{m} / \mathrm{s})$

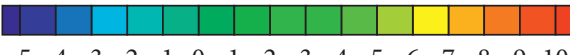

(c)

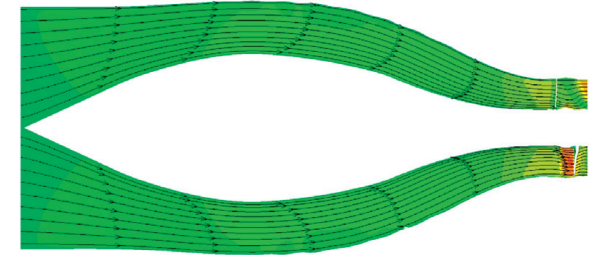

Axial Velocity $(\mathrm{m} / \mathrm{s})$

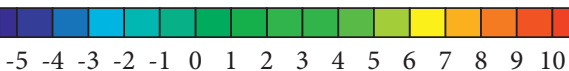

(b)

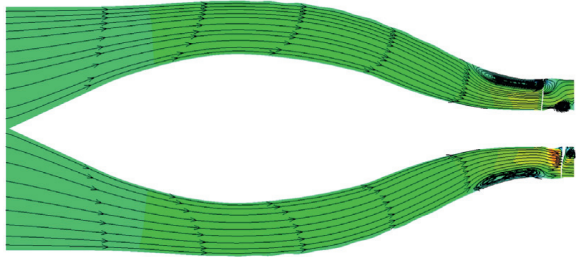

Axial Velocity $(\mathrm{m} / \mathrm{s})$

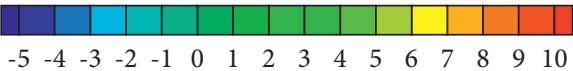

(d)

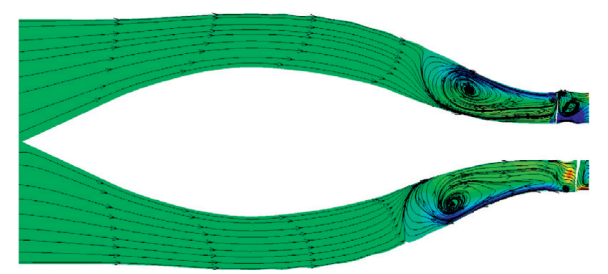

Axial Velocity $(\mathrm{m} / \mathrm{s})$

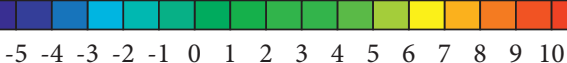

(e)

Figure 15: Internal flow pattern of the inlet passage under different conditions: (a) $Q / Q_{d}=1.0$; (b) $Q / Q_{d}=0.8$; (c) $Q / Q_{d}=0.6$; (d) $Q /$ $Q_{d}=0.32$; (e) $Q / Q_{d}=0.12$.

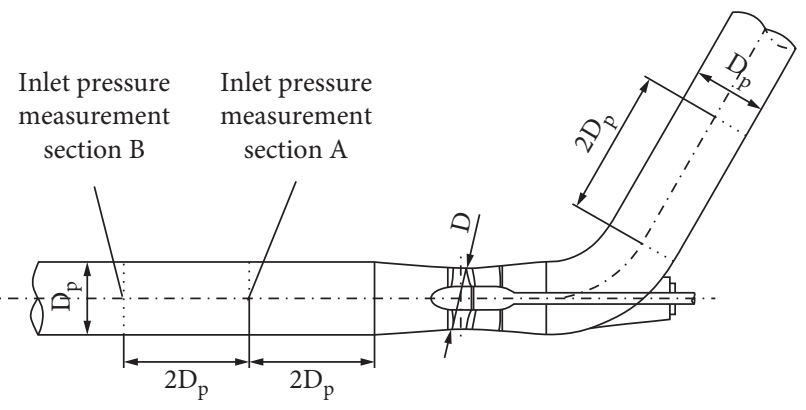

FIGURE 16: Location of different inlet pressure measurement sections.

higher. However, the pressure distribution on the inlet pressure measurement section $\mathrm{B}$ is uniform under all working conditions, and the pressure values at each point on the section are basically the same.

As shown in Figure 19, when the inlet pressure measurement section $\mathrm{A}$ is set at the same position as that in the model test, two saddle-shaped regions appear in the Q-H curve. However, when the inlet pressure measurement section is set at section $\mathrm{B}$, the $\mathrm{Q}-H$ curve has only one saddle-shaped region. This demonstrates that when the flow rate is less than $0.32 Q_{d}$, the influence range of backflow in the inlet pipe is large, which leads to the high-pressure zone near the wall of the inlet pressure measurement section, and hence the second saddle-shaped region of the axial-flow pump is essentially a measurement illusion. 


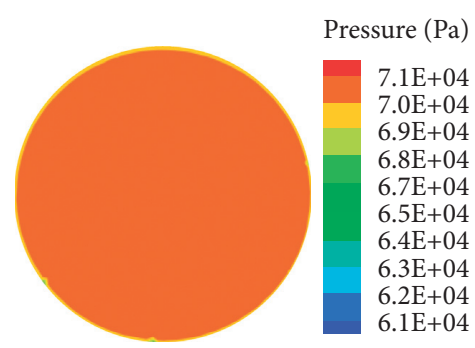

(a)

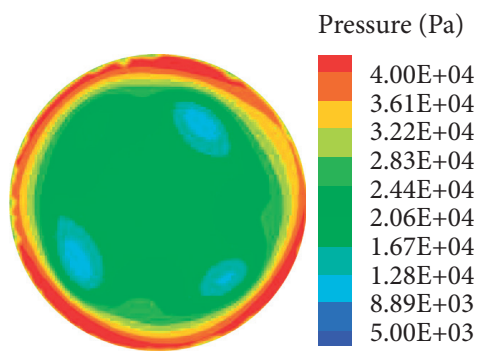

(d)

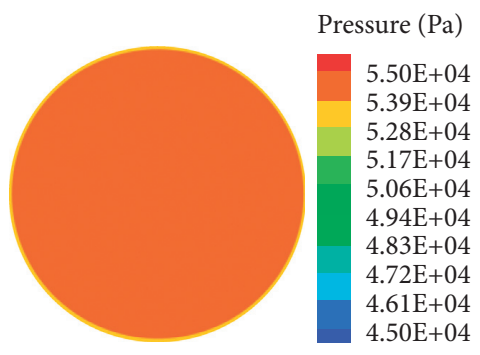

(b)

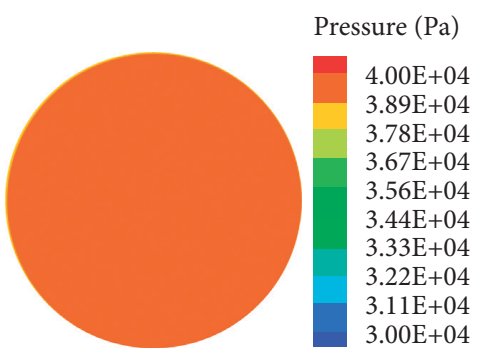

(c)

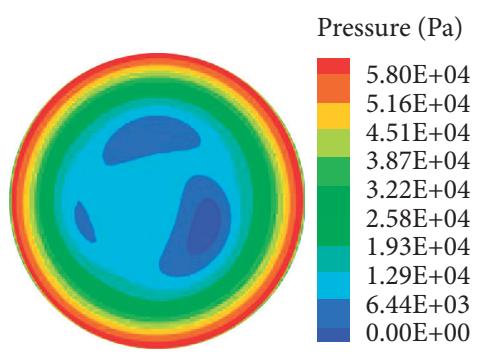

(e)

FiguRe 17: Pressure distribution of inlet pressure measurement section A: (a) $Q / Q_{d}=1.0$; (b) $Q / Q_{d}=0.80$; (c) $Q / Q_{d}=0.60$; (d) $Q / Q_{d}=0.32$; (e) $Q / Q_{d}=0.12$.

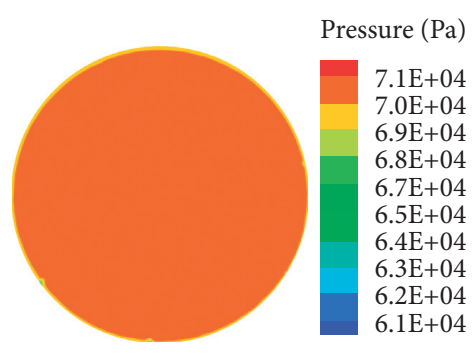

(a)

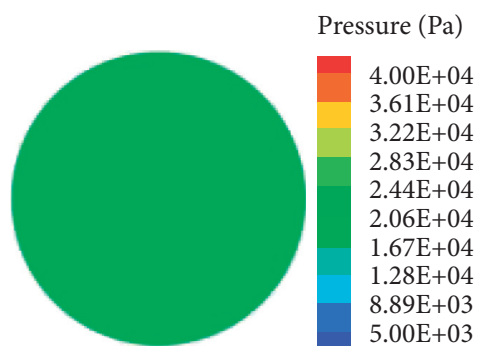

(d)

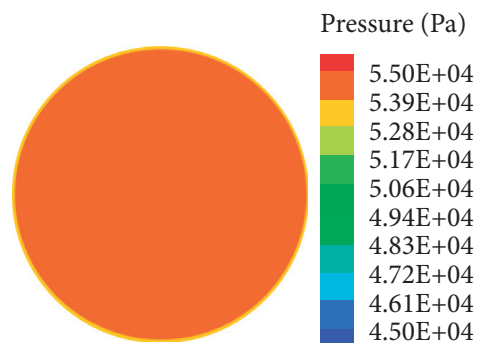

(b)

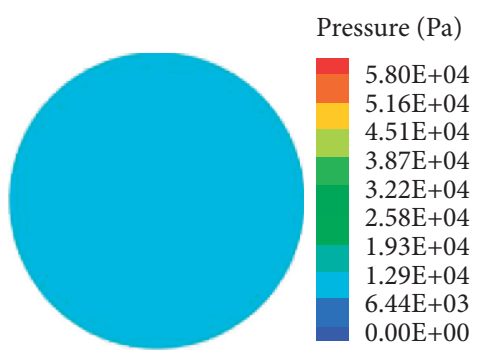

(e)

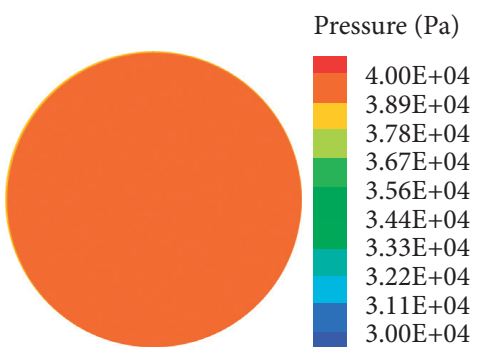

(c)

Figure 18: Pressure distribution of inlet pressure measurement section B: (a) $Q / Q_{d}=1.0$; (b) $Q / Q_{d}=0.80$; (c) $Q / Q_{d}=0.60$; (d) $Q / Q_{d}=0.32$; (e) $Q / Q_{d}=0.12$. 


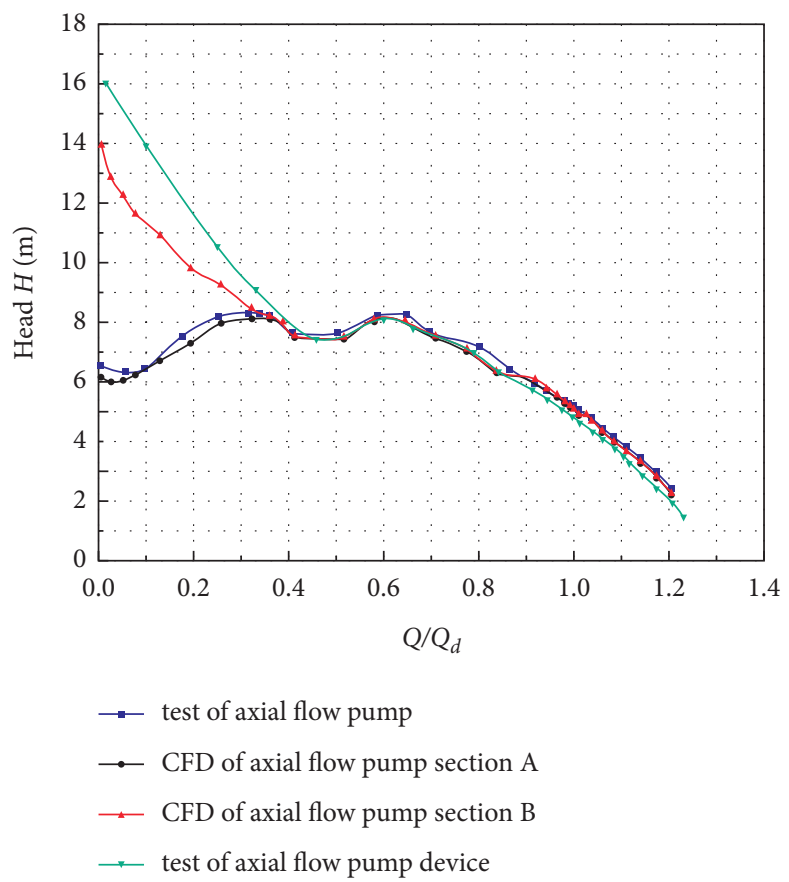

Figure 19: Comparison of different head-flow curves.

\section{Conclusions}

In this study, the energy performance tests of the TJ04-ZL-06 hydraulic model and its corresponding pump device were carried out to obtain the external curves, and numerical simulation was carried out. The conclusions are as follows:

(1) There are two saddle-shaped regions of the axial-flow pump, while there is only one saddle-shaped region of the corresponding device. The first saddle bottom head of the axial-flow pump is close to the saddle bottom head of the corresponding pump device, while the second saddle bottom head is obviously lower than the saddle bottom head of the corresponding pump device.

(2) The first saddle-shaped region of the axial-flow pump and the saddle-shaped region of the corresponding pump device are caused by the decrease of the lift coefficient due to the increase of the attack angle between flow and blade. The second saddleshaped region of the axial-flow pump is essentially a measurement illusion under small flow rate.

(3) It is suggested that the inlet pressure measurement section should be set at least $4 D_{p}$ away from the inlet flange of the impeller when testing the performance of the axial-flow pump with flow rate less than $0.32 Q_{d}$, which avoids the measurement illusion under small flow rate conditions.

(4) When selecting a pump for an axial-flow pumping station, the first saddle bottom head of the axial-flow pump or the saddle bottom head of the corresponding pump device should be taken as the control value of the highest head of the pumping station. The method makes up for the defect of the traditional method of choosing the control value and avoids the reduction of the operation range of the pumping station.

\section{Abbreviations}

$D: \quad$ Impeller diameter, $\mathrm{mm}$

$D_{p}: \quad$ Pipe diameter, $\mathrm{mm}$

Q: $\quad$ Flow, L/s

$Q_{d}: \quad$ Design flow, $\mathrm{L} / \mathrm{s}$

$n$ : $\quad$ Rotation speed, $\mathrm{r} / \mathrm{min}$

$v: \quad$ Inflow velocity, $\mathrm{m} / \mathrm{s}$

p: $\quad$ Flow field pressure, $\mathrm{Pa}$

$\rho: \quad$ Fluid density, $\mathrm{kg} / \mathrm{m}^{3}$

$\mu$ : $\quad$ Fluid dynamic viscosity, $\mathrm{m}^{2} / \mathrm{s}$

$G_{k}$ : The generation of turbulent kinetic energy

$\alpha_{k}$ : $\quad$ Prandtl numbers for $k$

$\alpha_{\varepsilon}: \quad$ Prandtl numbers for $\varepsilon$

$C_{1 \varepsilon}, \quad$ Empirical constants

$C_{1 \varepsilon}:$

H: Head, m

$P_{\text {out }}^{T}$ : Total pressure of the outlet pressure measurement section, $\mathrm{Pa}$

$P_{i n}^{T}: \quad$ Total pressure of the inlet pressure measurement section, $\mathrm{Pa}$

g: $\quad$ Local acceleration of gravity, $\mathrm{m} / \mathrm{s}^{2}$

$T_{p}: \quad$ Torque, $\mathrm{N} \cdot \mathrm{m}$.

\section{Data Availability}

The data in this paper were obtained by physical experiment and numerical simulation, and the data used to support the 
findings of this study are available from the corresponding author upon request.

\section{Conflicts of Interest}

The authors declare that they have no conflicts of interest regarding the publication of this paper.

\section{Authors' Contributions}

Dongtao Ji, Weigang Lu, and Lei Xu curated the data. Linguang Lu conducted formal analysis. Dongtao Ji wrote the original draft. Wei Shi, Jun Liu, and Ye Zhu reviewed and edited the article.

\section{Acknowledgments}

This study was supported by the National Natural Science Foundation of China (grant no. 51779215) and Water Conservancy Science and Technology Project of Jiangsu Province (grant no. 2019015).

\section{References}

[1] C. Liu, "Researches and developments of axial-flow pump system," Transactions of the Chinese Society for Agricultural Machinery, vol. 46, no. 6, pp. 49-59, 2015.

[2] L. Xu, L. G. Lu, W. Chen, and G. Wang, "Flow pattern analysis on inlet and outlet conduit of shaft tubular pump system of Pizhou pumping station in South-to-North water diversion project," Transactions of the Chinese Society of Agricultural Engineering, vol. 37, no. 10, pp. 653-658, 2012.

[3] D.-s. Zhang, W.-d. Shi, B. Chen, and X.-f. Guan, "Unsteady flow analysis and experimental investigation of axial-flow pump," Journal of Hydrodynamics, vol. 22, no. 1, pp. 35-43, 2010.

[4] L. Xu, L. G. Lu, W. Chen, and G. Wang, "Study on comparison of hydraulic design schemes for shaft tubular pump device," Journal of Hydroelectric Engineering, vol. 30, no. 5, pp. 207215, 2011.

[5] L. H. Xie, F. J. Wang, C. L. He, S. L. Su, S. C. Zhang, and B. L. Wang, "Experimental investigation on hydrodynamic characteristics of a 15 degree slanted axial-flow pump system," Journal of Hydraulic Engineering, vol. 50, no. 7, pp. 798-805, 2019.

[6] B. H. Wang, F. J. Wang, L. H. Xie, C. Y. Wang, S. L. Su, and Q. R. Zhu, "Flow deviation in the discharge passage of a slanted axial-flow pump system," Journal of Hydraulic Engineering, vol. 52, no. 7, pp. 829-840, 2021.

[7] J. Liu, Y. Zheng, D. Q. Zhou, Y. Mao, and L. Zhang, "Analysis of basic flow pattern in shaft front-positioned and shaft rearpositioned tubular pump systems," Transactions of the Chinese Society for Agricultural Machinery, vol. 41, pp. 32-38, 2010.

[8] R. Laborde, P. Chantrel, and M. Mory, "Tip clearance and tip vortex cavitation in an axial flow pump," Journal of Fluids Engineering, vol. 119, no. 3, pp. 680-685, 1997.

[9] Z. Li, M. G. Yang, N. Zhang, and B. Gao, "Experimental study on vibration characteristics of axial-flow pump under different operating conditions," Journal of Engineering and Thermophysics, vol. 34, no. 5, pp. 866-859, 2013.
[10] F. J. Wang, L. Zhang, and Z. M. Zhang, “Analysis on pressure fluctuation of unsteady flow in axial-flow pump," Journal of Hydraulic Engineering, vol. 38, no. 8, pp. 1003-1009, 2007.

[11] X. F. Guan, Manual for Selection of Large and Medium Low Head Pumps, China Machine Press, Beijing, China, 2019.

[12] H. Yang, D. D. Sun, F. P. Tang, and X. Zhang, "Experiment research on inlet flow field for axial-flow pump at unsteady operating condition," Journal of Drainage and Irrigation Machinery Engineering, vol. 29, no. 5, pp. 406-410, 2011.

[13] Y. Zheng, Y. T. Mao, D. Q. Zhou, and D. Zhang, "Flow characteristics of low-lift and large flow rate pump installation in saddle zone," Journal of Drainage and Irrigation Machinery Engineering, vol. 29, no. 5, pp. 369-373, 2011.

[14] I. Goltz, G. Kosyna, U. Stark, H. Saathoff, and S. Bross, "Stall inception phenomena in a single-stage axial-flow pump," Proceedings of the Institution of Mechanical Engineers-Part A: Journal of Power and Energy, vol. 217, no. 4, pp. 471-479, 2003.

[15] Q. C. Zheng, W. M. Feng, L. C. Zhou, and N. Li, "Effects of inlet guide vane on characteristics of backflow vortex in hump region of axial flow pump," Transactions of the Chinese Society for Agricultural Machinery, vol. 47, no. 4, pp. 8-14, 2016.

[16] Y. Wang, N. C. He, X. F. Wu, H. L. Liu, and L. Zhang, "Inner flow characteristics analysis of axial flow pump in the saddle zone," China Rural Water and Hydropower, vol. 11, pp. 164-167, 2017.

[17] L. G. Lu, Optimal Hydraulic Design of High Performance Large Low Head Pump System, China Water Power Press, Beijing, China, 2013.

[18] L. Shi, W. Zhang, H. Jiao et al., "Numerical simulation and experimental study on the comparison of the hydraulic characteristics of an axial-flow pump and a full tubular pump," Renewable Energy, vol. 153, pp. 1455-1464, 2020.

[19] H. Zhang, W. D. Shi, B. Chen, Q. H. Zhang, and W. D. Cao, "Experimental study of flow field in interface region between impeller and guide vane of axial flow pump," Journal of Hydrodynamics, vol. 26, no. 6, pp. 894-901, 2015.

[20] W. Chen, L. G. Lu, G. Wang, and L. Dong, "Influence of axialflow pump operating condition on the pre-swirl at impeller chamber inlet," Journal of Hydroelectric Engineering, vol. 31, no. 1, pp. 213-219+225, 2012, in Chinese.

[21] C. Xie, F. Tang, R. Zhang, W. Zhou, W. Zhang, and F. Yang, "Numerical calculation of axial-flow pump's pressure fluctuation and model test analysis," Advances in Mechanical Engineering, vol. 10, no. 4, pp. 1-13, 2018.

[22] L. J. Shi, F. P. Tang, H. L. Zhou, and L. Tu, "Axial-flow pump hydraulic analysis and experiment under different sweptangles of guide vane," Transactions of the Chinese Society of Agricultural Engineering, vol. 31, pp. 90-95, 2015.

[23] X. Guo, Z. Zhu, B. Cui, and G. Shi, "Effects of the number of inducer blades on the anti-cavitation characteristics and external performance of a centrifugal pump," Journal of $\mathrm{Me}$ chanical Science and Technology, vol. 30, no. 7, pp. 3173-3181, 2016.

[24] J. Jiang, Y.-h. Li, C.-y. Pei et al., "Cavitation performance of high-speed centrifugal pump with annular jet and inducer at different temperatures and void fractions," Journal of $\mathrm{Hy}$ drodynamics, vol. 31, no. 1, pp. 93-101, 2019.

[25] Y. Q. Wang, H. D. Zhang, Z. B. Han, and X. Ni, “Optimization design of centrifugal pump flow control system based on adaptive control," Processes, vol. 9, no. 9, 2019.

[26] W. Wan and W. Huang, "Investigation on complete characteristics and hydraulic transient of centrifugal pump," Journal of Mechanical Science and Technology, vol. 25, no. 10, pp. 2583-2590, 2011. 
[27] M. Liu, L. Tan, and S. Cao, "Method of dynamic mode decomposition and reconstruction with application to a threestage multiphase pump," Energy, vol. 208, Article ID 118343, 2020.

[28] M. Liu, L. Tan, Y. Xu, and S. Cao, "Optimization design method of multi-stage multiphase pump based on Oseen vortex," Journal of Petroleum Science and Engineering, vol. 184, Article ID 106532, 2020.

[29] C. Wang, X. Chen, N. Qiu, Y. Zhu, and W. Shi, "Numerical and experimental study on the pressure fluctuation, vibration, and noise of multistage pump with radial diffuser," Journal of the Brazilian Society of Mechanical Sciences and Engineering, vol. 40 , no. 10 , p. $481,2018$.

[30] M. Liu, L. Tan, and S. Cao, "Theoretical model of energy performance prediction and BEP determination for centrifugal pump as turbine," Energy, vol. 172, pp. 712-732, 2019.

[31] B. Jafarzadeh, A. Hajari, M. M. Alishahi, and M. H. Akbari, "The flow simulation of a low-specific-speed high-speed centrifugal pump," Applied Mathematical Modelling, vol. 35, no. 1, pp. 242-249, 2011.

[32] F. J. Wang, Analysis Method of Flow in Pumps and Pumping Stations, China Water Power Press, Beijing, China, 2019.

[33] V. Yakhot and S. A. Orszag, "Renormalization group analysis of turbulence. I. Basic theory," Journal of Scientific Computing, vol. 1, no. 1, pp. 3-51, 1986.

[34] L. Cheng, C. Liu, F. P. Tang, and J. Zhou, "3D numerical simulation and performance predication of vertical axial flow pumping station by RNG turbulent model," Journal of Mechanical Engineering, vol. 45, no. 3, pp. 252-257, 2009.

[35] Q. G. Chen, Z. Xu, and Y. J. Zhang, "Application of RNG k-E models in numerical simulation of engineering turbulent flows," Chinese Quarterly of Mechanics, vol. 24, pp. 88-95, 2003.

[36] Z. H. Xu, Y. L. Wu, N. X. Chen, Y. Liu, L. Liang, and Y. Z. Wu, "Simulation of turbulent flow in pump based on sliding mesh and RNG k-E model," Journal of Engineering Thermophyiscs, vol. 26, pp. 66-68, 2005.

[37] Y. J. Zheng, K. Wang, X. C. Lei, and F. Tang, "3D numerical simulation in inlet passages of pumping station by RNG $\mathrm{k}-\varepsilon$ turbulent model with wall-function law," Water Resources and Power, vol. 26, pp. 123-125, 2008.

[38] W. C. Zierke and W. A. Straka, "Flow visualization and the three-dimensional flow in an axial-flow pump," Journal of Propulsion and Power, vol. 12, no. 2, pp. 250-259, 1996.

[39] D. S. Zhang, L. Shi, J. Chen, Q. Pan, and W. D. Shi, "Experimental analysis on characteristic of cavitation in tip region of axial flow pump impeller," Journal of Zhejiang University (Engineering Science), vol. 8, pp. 1585-1592, 2016.

[40] D. Zhang, L. Shi, W. Shi, R. Zhao, H. Wang, and B. P. M. van Esch, "Numerical analysis of unsteady tip leakage vortex cavitation cloud and unstable suction-side-perpendicular cavitating vortices in an axial flow pump," International Journal of Multiphase Flow, vol. 77, pp. 244-259, 2015. 